\title{
Correction to: Influence of Gd-EOB-DTPA on proton density fat fraction using the six-echo Dixon method in 3 Tesla magnetic resonance imaging
}

Tatsuya Hayashi ${ }^{1} \cdot$ Kei Fukuzawa ${ }^{2} \cdot$ Hiroshi Kondo ${ }^{3} \cdot$ Hiroshi Onodera $^{4} \cdot$ Shuji Toyotaka $^{4} \cdot$ Rie Tojo $^{4}$. Shimpei Yano $^{4} \cdot$ Masakatsu Tano $^{2} \cdot$ Tosiaki Miyati $^{5} \cdot$ Jun'ichi Kotoku $^{1} \cdot$ Takahide Okamoto $^{1} \cdot$ Keiko Toyoda ${ }^{3} \cdot$ Hiroshi Oba ${ }^{3}$

Published online: 14 March 2018

(C) Japanese Society of Radiological Technology and Japan Society of Medical Physics 2018

Correction to: Radiol Phys Technol (2017) 10:483-488 https://doi.org/10.1007/s12194-017-0420-7

A diameter of glass bottles in phantoms in the above article $(2 \mathrm{~cm})$ was incorrect. The correct diameter is $4.5 \mathrm{~cm}$.

The original article can be found online at https:// doi.org/10.1007/s12194-017-0420-7.

Tatsuya Hayashi

t-hayashi@med.teikyo-u.ac.jp

1 Graduate School of Medical Technology, Teikyo University, 2-11-1 Kaga, Itabashi-ku, Tokyo 173-8605, Japan

2 Department of Radiological Technology, Toranomon Hospital, Tokyo, Japan

3 Department of Radiology, Teikyo University School of Medicine, Tokyo, Japan

4 Central of Radiology, Teikyo University Hospital, Tokyo, Japan

5 Faculty of Health Sciences, Institute of Medical, Pharmaceutical and Health Sciences, Kanazawa University, Ishikawa, Japan 